\title{
Comparative evaluation of two porcine ex vivo models for training in endoscopic ultrasound-guided drainage of pancreatic fluid collections $\square$
}

\section{(ㄷ)(1) $(2) \Theta$}

Authors

Frederick Moryoussef ${ }^{1}$, Sarah Leblanc ${ }^{2}$, Alice Bertucat ${ }^{3}$, Arthur Laquiere ${ }^{4}$, Emmanuel Coron ${ }^{5}$, Luigi Mangialavori² ${ }^{2}$ Jean-Christophe Duchmann ${ }^{6}$, Yann Le Baleur ${ }^{7}$, Frederic Prat $^{2}$

Institutions

1 Department of Gastroenterology, La Pitié Salpetrière Teaching Hospital, AP-HP, University Pierre et Marie Curie, Paris, France

2 Department of Gastroenterology, Cochin Teaching Hospital, AP-HP, University Paris Descartes, Sorbonne Paris Cite, Paris, France

3 Life Partners Europe, 161, Avenue Gallieni, 93170 Bagnolet, France

4 Department of Gastroenterology and Hepatology, Saint Joseph Hospital, Marseille, France

5 Department of Gastroenterology and Hepatology, Nantes Teaching Hospital, Nantes, France

6 Department of Gastroenterology and Hepatology, General Hospital of Compiegne, Compiègne, France

7 Department of Gastroenterology, Henri Mondor Teaching Hospital, AP-HP, University Paris Est Creteil, Creteil, France

submitted 6.2.2017

accepted after revision 2.5.2017

\section{Bibliography}

DOI https://doi.org/10.1055/s-0043-117945 |

Endoscopy International Open 2017; 05: E1020-E1026

(c) Georg Thieme Verlag KG Stuttgart · New York ISSN 2364-3722

Corresponding author

Frederic Prat, MD, PhD, Department of Gastroenterology, Cochin Teaching Hospital, AP-HP, University Paris

Descartes, Sorbonne Paris Cite, Paris, France

Fax: +33-1-58411965

frederic.prat@aphp.fr

\section{ABSTRACT}

Introduction EUS-guided cystoenterostomy (EUCE), a technique used for the drainage of pancreatic pseudocysts and peri-enteric collections, requires specific skills for which dedicated models are needed. Based on a compact EASIE model (Erlangen Active Simulator for Interventional Endoscopy), we developed two ex vivo porcine models of retrogastric cysts and evaluated learning performance within the frame of a structured training program.

Material and methods The first model was made of porcine colon (i.e. "natural cyst"), the second one with an ostomy bag (i.e. "artificial cyst"). All procedures were achieved with an EUS scope under fluoroscopy. Both models were evaluated prospectively over a 2-day session involving 14 students and five experts. The primary end point was overall satisfaction with each model.

Results The "natural cyst" and "artificial cyst" were prepared within 10 and 16.5 minutes $(P=0.78)$, respectively. Model grading showed a non-significant trend for overall satisfaction in favor of the artificial model $(P=0.06)$. As secondary end points, difference was not significant for impression of realism $(P=0.75)$ whereas the "artificial cyst" was graded significantly better by experts and students in terms of ability to teach procedural steps $(P=0.01)$ and ease of puncture $(P=0.03)$. Moreover, experts considered the ability to improve students' proficiency to be superior with the "artificial cyst" $(P=0.008)$.

Conclusion Both "artificial" and "natural cysts" are efficient for EUCE training in terms of overall satisfaction. However, the "artificial cyst" model appears to make the procedure easier with a higher ability to teach procedural steps and improve the students' proficiency. Larger applications of this model are needed to validate as a standard of training.

\section{Introduction}

Pancreatic fluid collections develop in $5-16 \%$ of patients following acute pancreatitis, in up to $40 \%$ of chronic pancreatitis patients, as well as in some patients after pancreatic surgery or pancreatic trauma [1,2]. Indications for drainage include symptomatic collections (with pain or obstruction of the gastric outlet or biliary tract) and infected collections or walled-off pancreatic necrosis [3]. Other peri-enteric, non-pancreatic, collections may also require drainage, such as certain peri-rectal 
abscesses [4]. The endoscopic transmural technique has gained wide acceptance for the drainage of pancreatic pseudocysts and likewise peri-enteric collections as a less invasive alternative to surgical and percutaneous drainage, with lower morbidity and more rapid recovery [5].

Endoscopic ultrasound (EUS) guidance has significantly extended the reach of endoscopic drainage, and improved the success rate and safety thanks to a lower rate of hemorrhage and perforation [3]. EUS-guided cystoenterostomy (EUCE) has subsequently become a widespread interventional technique. However, EUS-endoscopic drainage remains a complex technique with a mean overall complication rate of $15 \%$ [3]. Some authors have claimed that a minimum number of 20 expert-supervised procedures should be performed in a tertiary referral center to guarantee proficiency, which corresponds to the upper limit of the mean annual caseload in most expert centers $[6,7]$. It appears therefore necessary to train endoscopists in a preclinical setting to shorten the learning curve and reduce the number of expert-supervised clinical cases needed to attain proficiency in EUCE. The present study was undertaken to evaluate the efficiency of newly developed animal models for EUCE lab training.

\section{Materials and methods}

\section{Study setting}

Gastroenterologists willing to specialize in interventional endoscopy in France are encouraged to undergo a 2-year structured training program which includes theoretical sessions, clinical training in countrywide-selected expert centers, and several animal workshops on specific techniques (endoscopic retrograde cholangiopancreatography [ERCP], endoscopic submucosal dissections [ESD], etc.) held at the Assistance Publique Hôpitaux de Paris School of Surgery. After a careful selection of candidates and a successful final theoretic and practical examination, trainees are certified in digestive and biliopancreatic interventional endoscopy. The present study took place in April 2016 during one of the animal workshops dedicated to biliopancreatic endoscopy for second-year students (gastroenterology fellowship with basic EUS diagnosis training).

\section{Animal models}

Models were designed and tested a few weeks before formal student training. Animal material came from a slaughterhouse in the Paris area (Saint Mathieu le Moulin, 78550, France), which was veterinary controlled and authorized for experimental use. Models used in the study were derived from the Compact EASIE model (Erlangen Active Simulator for Interventional Endoscopy) and prepared at the School of Surgery (8/10 rue des Fossés saint Marcel, Paris, 75005, France) on the same day as student training by assistants specially trained in the preparation of various surgical and endoscopic models.

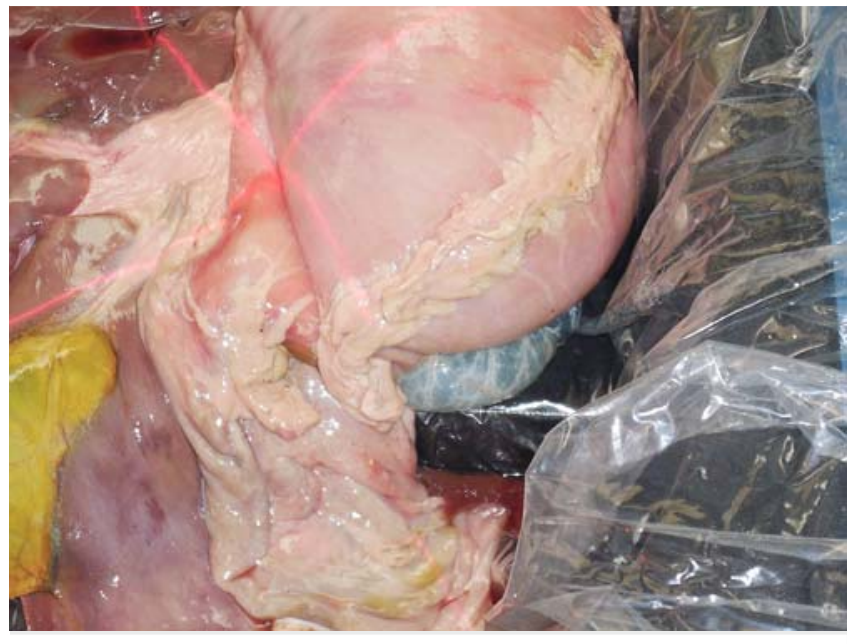

- Fig. 1 "Natural cyst" (below stomach) before drainage.

\section{Model preparation procedure}

\section{Compact EASIE model}

The Compact EASIE model was described initially by Hochberger and the Erlangen team and was shown to be a valuable tool for teaching endoscopic skills and techniques [8-10]. In our EUCE models, the proximal digestive tract $(25-30 \mathrm{~cm}$ of esophagus, stomach, and the whole duodenum) was collected en bloc from the slaughterhouse with or without the liver and biliary tract. The stomach was carefully washed using a small gastrotomy and pieces were frozen before delivery. Once thawed, the organ bloc was fixed to a standard X-ray compatible set-up with several stitches, the esophagus being attached to a plastic inlet for scope insertion. A patient plate for monopolar current was placed under the stomach to allow for electrocautery.

\section{Model \#1: the "natural cyst"}

The first model, the so-called "natural cyst", was derived from a report by Baron et al. using native porcine tissue to create a fluid collection [11]. We used colon instead of small intestine, preparing $10-20 \mathrm{~cm}$ long sections after careful rinsing and one-sided suturing, before filling those segments with a mixture of ultrasound gel and water and suturing the other side. This "pouch" was subsequently sutured to the posterior gastric wall and covered by the liver in order to ensure realistic EUS imaging and create some pressure over the collection during EUCE ( Fig. 1).

\section{Model \#2: the "artificial cyst"}

In this model, we used a digestive bloc with or without liver and bile ducts and a two piece transparent ostomy (skin barrier with flange and pouch - for example, Hollister $580 \mathrm{ml}$ ref., Hollister ref. 25850, Hollister Inc., Libertyville, IL, United States). The skin barrier was stitched to the gastric wall using cotton gauze to ensure watertightness (internal sticthes, cotton gauze then external stitches), before filling the pouch with the desired amount of ultrasound gel. Once completed, we closed the ost- 

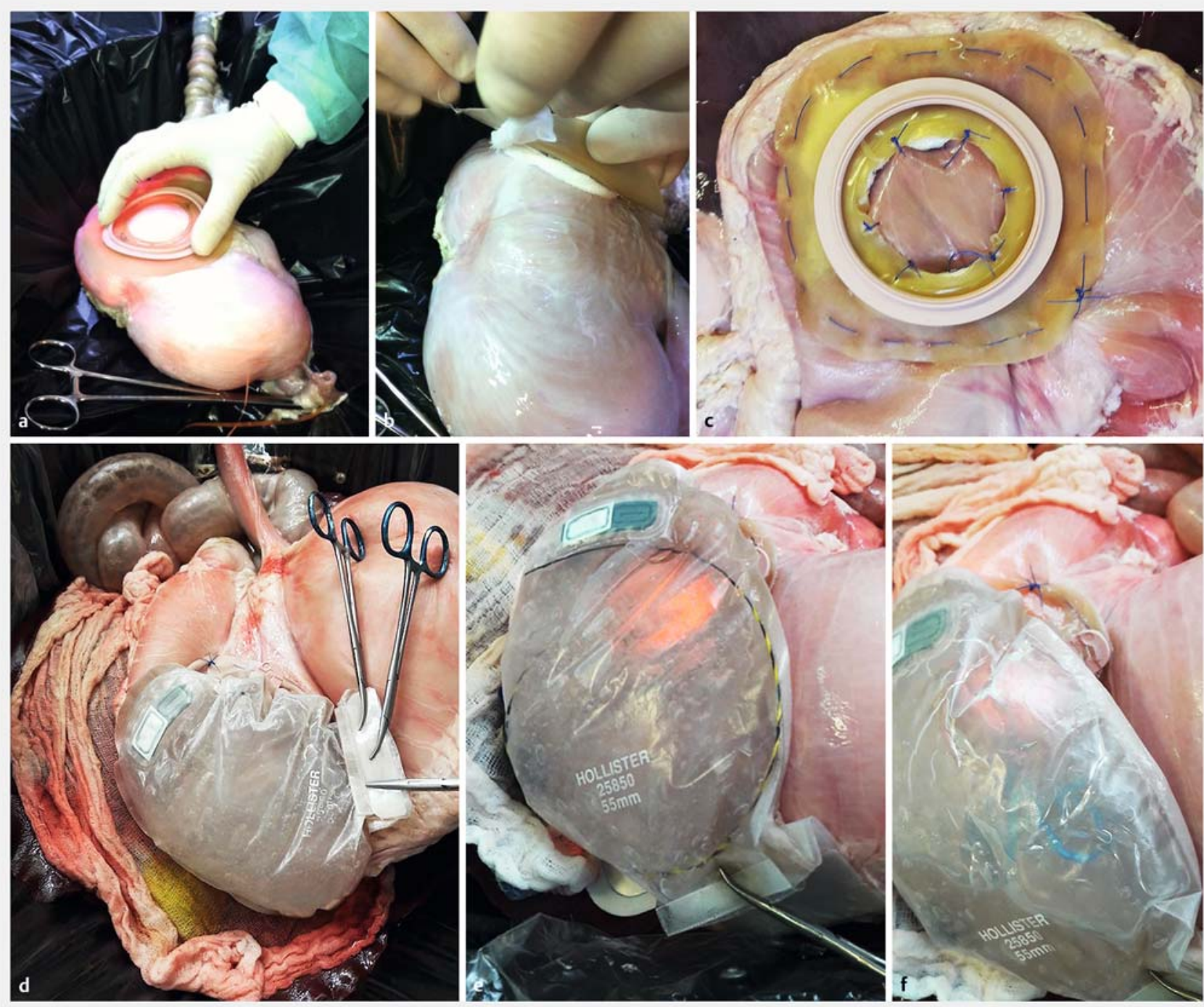

- Fig. 2 a-d "Artificial cyst". An ostomy bag is stitched to the posterior gastric wall using cotton gauze to ensure water tightness. The ostomy bag is subsequently filled with ultrasound gel. $\mathbf{e}-\mathbf{f}$ Guidewire and double pigtail stents are seen in the ostomy bag.

omy with clamps to maintain tightness during training sessions ( Fig.2a-d).

\section{EUCE procedure}

Procedures were achieved under fluoroscopy and using a curved linear array EUS-scope (GF-UCT 140 and EU-M2 ultrasound platform, Olympus, Tokyo, Japan). The collection or "cyst" was identified from the gastric body and punctured with a 19-gauge needle (Expect, Boston Scientific S.A. (Pty) Ltd) ( $\vee$ Video 1$)$, before contrast injection (optional), and pushing of a 0.035 -inch guidewire (Jagwire; Boston Scientific, Natick, MA, United States) into the cyst. The needle was removed, and the track was coagulated using a $6 \mathrm{Fr}$ cystotome (Endoflex, Brussels, Belgium) before the insertion of a $7 \mathrm{Fr}, 5 \mathrm{~cm}$ long double pigtail plastic stent (Advanix, Boston Scientific). When needed for stent insertion or for training purposes, balloon dilatation could be performed ( $8-12 \mathrm{~mm}$ large Hurricane or CRE balloon, Boston Scientific) ( $\vee$ Video 2 ).

\section{Study design}

Once a model was ready for use, it was first tested by its designers (AB, SL, FP) for its ability to allow EUCE procedures. Five experts otherwise involved in onsite ERCP workshops (AL, EC, JCD, $L M, Y L B$ ) were subsequently asked to perform a procedure themselves and then to supervise a group of second year students during a procedure. All of the experts had more than 5 years of experience in interventional EUS and pseudocyst drainage and estimated their own number of previous EUCE procedures between 20 and more than 60 patients. Each student had to perform two procedures on only one of the two models ("natural cyst" or "artificial cyst") over the 2-day training course. Two models of each type were used. Experts and students had to complete a written form before the procedure for pre-test criteria and immediately after performing EUCE for post-test criteria. 


\section{Study end points}

The main end point was global satisfaction (for experts and students) with the model as a training tool (defined as the ability of the model to allow for constant interaction between expert and trainee).

Experts and/or students were also asked to grade each model for the subsequent end points (on an analogue 10-point scale for semi-quantitative criteria):

Secondary end points are listed below:

1. Time for preparation of the model.

2. Ability of the model to behave realistically and to teach all the procedural steps.

3. Overall impression of realism, defined as conformity with actual images and cognitive experience during a procedure in a patient.

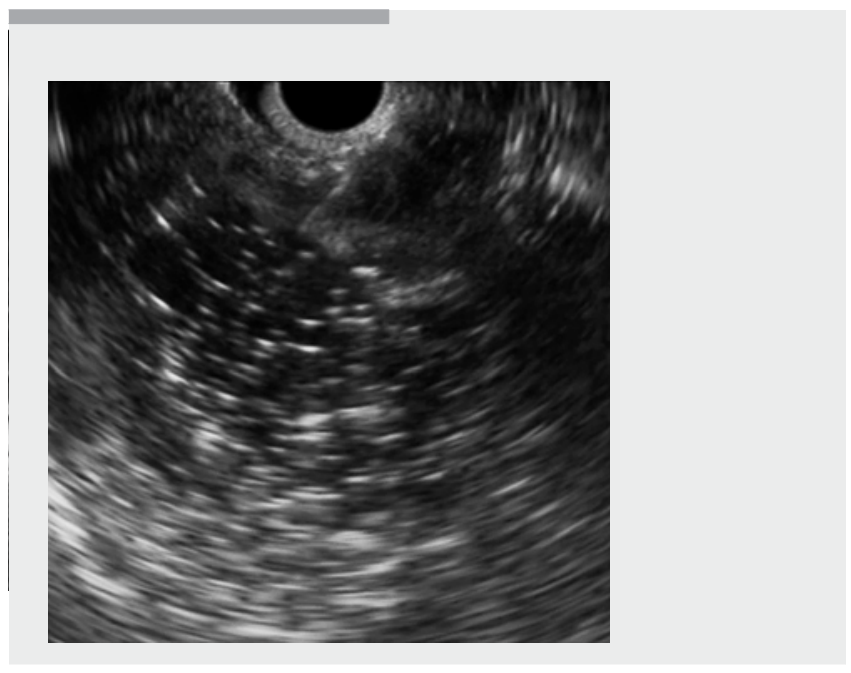

$\checkmark$ Video 1 Puncture of "natural cyst” under EUS guidance.

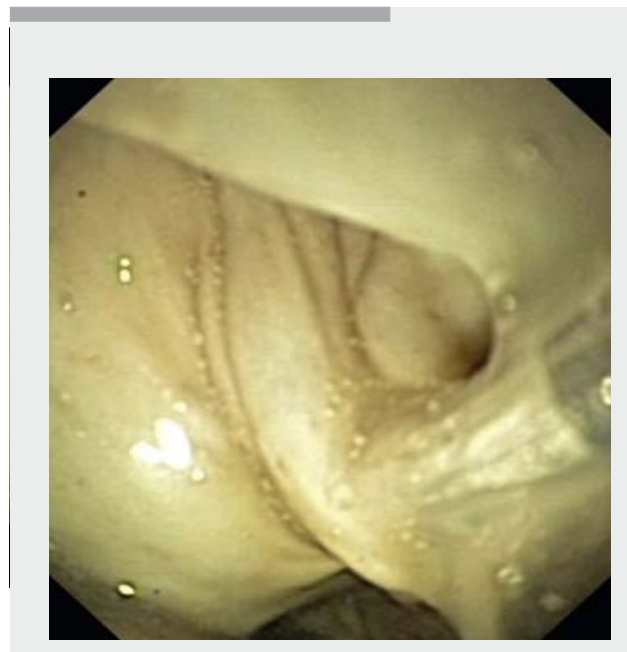

Video 2 Balloon dilatation after puncture. Ultrasound gel is seen under direct vision.
4. Ease of puncture.

5. Ability to increase student proficiency (experts only).

6. Improvement in self-confidence for human procedure (students only with pre- and post-test).

7. Improvement in individual skills (perception of self-improvement - students only with pre- and post-test).

8. Time for procedure (students only).

9. Procedure success (students only).

\section{Statistical analysis}

Quantitative variables are expressed as means (standard deviation; SD) and qualitative data as percentages (\%). The Student's $t$ test (for continuous variables) or Fisher's exact test (for qualitative variables) were used. The primary end point was evaluated for (a) students, (b) experts, and (c) students and experts combined. A $P$ value $<0.05$ defined statistical significance. All statistical tests were computed with BiostaTGV (http://marne. u707.jussieu.fr/biostatgv/).

\section{Results}

Time for preparation of "natural cysts" and "artificial cysts" was $10 \pm 0.5$ and $16.5 \pm 1$ minutes, respectively $(P=0.78)$. Between 10 and 13 EUCE procedures could be achieved on each model and the number of EUS-guided punctures was not a limitation in any of the models.

\section{Evaluation}

Evaluations are displayed in $>$ Table 1. No statistically significant difference was observed in terms of overall satisfaction (primary end point). Regarding secondary end points for both groups, no difference was observed for overall impression of realism (Students: $P=0.84$, Experts: $P=0.6$; All $P=0.75$ ). Nevertheless, significant differences in pooled groups favoring the "artificial cyst" were observed in terms of ability to teach procedure steps (Students: $P=0.18$; Experts: $P=0.42$; All: $P=0.01$ ) and ease of puncture (Students: $P=0.19$; Experts: $P=0.4$; All: $P=$ 0.03). Indeed, two experts and three students complained about a difficult puncture of the "natural cyst" because of its elasticity, requiring several attempts to puncture both the gastric and the "cystic" walls ( $\triangleright$ Video 1, $>$ Table 2 ), whereas none of the experts and students using the artificial model complained about that point. Moreover, the "artificial cyst" model was granted a better score than the "natural cyst" one for its ability to increase student proficiency $(P=0.008)$ when only experts evaluated it; however, there were no statistically significant differences for improvement in self-confidence for human procedures $(P=0.73)$ and in individual skills $(P=0.33)$ when students evaluated both models. No statistically significant difference was observed for procedure time $(P=0.78)$ and success of the procedure $(P=1)$.

\section{Additional notes}

Another advantage of the "artificial cyst" model was the possibility to work under fluoroscopy by hiding the ostomy bag under a tissue sheet, but also to remove the sheet and watch the guidewire through the transparent bag, thus helping students 
- Table 1 Comparative results between groups ("artificial cyst" versus "natural cyst" evaluated by students and experts with regard to primary and secondary end points).

\begin{tabular}{|c|c|c|c|}
\hline & “Artificial cyst” group & “Natural cyst" group & $P$ value \\
\hline \multicolumn{4}{|l|}{ Global satisfaction, mean (SD) ${ }^{1}$} \\
\hline - Students & $8.4(1)$ & $7.6(1.4)$ & 0.21 \\
\hline - Experts & $7.5(0.7)$ & $4.7(2.1)$ & 0.13 \\
\hline - All & $8.2(1)$ & $6.7(2.1)$ & 0.06 \\
\hline \multicolumn{4}{|l|}{ Ability of the model to teach steps, mean (SD) ${ }^{1}$} \\
\hline - Students & $7.4(1.3)$ & $6.3(1.7)$ & 0.18 \\
\hline - Experts & $8(0)$ & $6.6(2.3)$ & 0.42 \\
\hline - All & $7.5(1.2)$ & $5.9(1.7)$ & 0.01 \\
\hline \multicolumn{4}{|l|}{ Impression of realism, mean (SD) ${ }^{1}$} \\
\hline - Students & $7.3(0.76)$ & $7.4(1.6)$ & 0.84 \\
\hline - Experts & $6.5(0.7)$ & $5.7(2.3)$ & 0.6 \\
\hline - All & $7.1(0.8)$ & $6.9(1.9)$ & 0.75 \\
\hline \multicolumn{4}{|l|}{ Ease of puncture (number grading puncture as "difficult"), $n$ (\%) } \\
\hline - Students & 0 & $3(42.8)$ & 0.19 \\
\hline - Experts & 0 & $2(66.7)$ & 0.4 \\
\hline - All & 0 & $5(50)$ & 0.03 \\
\hline Ability to increase student proficiency, mean (SD) ${ }^{1}$ (Experts only) & $8(0)$ & $4.3(0.57)$ & 0.008 \\
\hline $\begin{array}{l}\text { Improvement (\%) in self confidence for human procedure, mean (SD) }{ }^{1} \\
\text { (Students only) }\end{array}$ & $212.4(203.8)$ & $214.3(184.4)$ & 0.73 \\
\hline Improvement (\%) in individual skills, mean (SD) ${ }^{1}$ (Students only) & $200.3(202.4)$ & $99.5(140.3)$ & 0.33 \\
\hline Time of procedure, mean (SD), min (Students only) & $10(0.5)$ & $16.5(1)$ & 0.78 \\
\hline Success of procedure, $n$ (\%) (Students only) & $6(85.7)$ & $5(71.4)$ & 1 \\
\hline
\end{tabular}

understand guidewire actuation and control ( $>$ Fig. 2 e $-\mathbf{f},>$ Table2). Negative comments by one expert and two students with regard to both techniques concerned ultrasound gel containing microbubbles making for an inhomogeneous echogenicity of the collection and impairing needle recognition.

We valued the "artificial" and "natural cyst" models at, respectively 70 and 60 euro, tax included. These costs include organ preparation and delivery (including the liver and biliary tract in the "natural cyst" model, as well as ancillary material in the "artificial cyst" model (ostomy bag, sutures, etc.)). The purchase of an "Easie Model" set-up (2600 euro), which can be reused indefinitely for training purposes in endoscopy, is also to be recommended for both models.

\section{Discussion}

Endoscopic drainage of peri-enteric collections, when guided by EUS, as is nowadays commonplace and recommended, requires the acquisition of specific skills over a learning curve. This technique is performed either by biliary endoscopists trained in catheter exchange and stent insertion, but not in ultrasound imaging and fine needle aspiration (FNA), or by endosonographers proficient in needle manipulation but not in elementary interventional procedures. Specific training courses on validated models are necessary to achieve efficient and safe patient procedures.

Two different animal models have been described in the past. The first, published in 2006 by Schöfl et al., used the gallbladder of an EASIE model as a simulator for pseudocyst drainage, but the small volume of the gallbladder and rapid bile leakage after puncture precluded its use by several consecutive trainees [7]. In 2009, but published in 2015, using the porcine sigmoid colon as a "natural cyst" and a porcine stomach positioned in a stainless steel pan, Baron et al. showed that their model was reliable for cystoenterostomy as well as for endoscopic necrosectomy and reported many applications of the model in live demonstrations and workshops [11]. Basically, this model was similar to the "natural cyst" described in this report, apart from the significant addition of latex tubes connected to a peristaltic pump in order to simulate vessels and blood 
- Table2 Advantages and drawbacks of "natural cyst" versus "artificial cyst".

\begin{tabular}{|c|c|c|}
\hline & “Natural cyst” & "Artificial cyst" \\
\hline \multirow[t]{4}{*}{ Advantages } & Biological & Only one wall to pass (gastric) \\
\hline & Shorter time for preparation of cysts & Direct guidewire visualization in addition to fluoroscopy \\
\hline & Other EUS procedures feasible (FNA, biliary drainage) & Direct modification of cyst content \\
\hline & & Better at teaching procedure steps and improving proficiency \\
\hline \multirow[t]{3}{*}{ Drawbacks } & Elasticity $=$ puncture more difficult & Longer time for preparation \\
\hline & No direct view of guidewire (fluoroscopy only) & $\begin{array}{l}\text { Not amenable to other interventional EUS procedures } \\
\text { (e.g. EUS-guided biliary drainage) }\end{array}$ \\
\hline & $\begin{array}{l}\text { No straightforward modification of cyst content during training } \\
\text { (but different cysts can be exchanged easily) }\end{array}$ & \\
\hline
\end{tabular}

flow. This study did not intend to complicate model preparation, but "blood vessels" can easily be added to the "natural cyst" as is commonly done for hemostasis workshops using the EASIE model $[9,10]$. It would be a little more difficult with the "artificial cyst" but this difficulty could easily be overcome by inserting the vessel tubes in the gastric submucosa. We plan to introduce this feature in our model for future workshops.

Model preparation time is an important issue when organizing a workshop with a relatively large number of participants. Model preparation in Baron's model required 20-30 minutes, which was double that of our "natural cyst" but only a little longer than the "artificial cyst", which can be explained by the absence of vessels in our study. Baron's model had a good rating score from experts and allowed for numerous procedures, similar to our findings. However, the steel pan did not allow for fluoroscopy, which, although optional, is useful for a full learning of drainage techniques. Moreover, this model had not been fully evaluated in a group of students. The structured format offered by our interventional endoscopy workshops allowed us to try to improve Baron's model by implementing fluoroscopy, to undertake a formalized assessment of the model, and to explore another method to emulate EUCE, using an ostomy bag instead of natural tissue.

Our main findings were that both models were able to teach EUCE to interventional endoscopy trainees with equal satisfaction under realistic conditions, although with a non-significant trend in favor of the artificial model, which additionally appeared to make puncture significantly easier and allowed better teaching of procedural steps ( $\triangleright$ Table 2 ).

Regarding the experts' opinion on the ability of the model to improve trainees' proficiency, which significantly favored the "artificial cyst" model, this was partly due to the visibility of the guidewire through the ostomy bag, making it easier for the trainer to explain and for the trainee to understand guidewire control during EUCE. Moreover, cyst size can be reduced (using smaller volumes or by folding the bag) when desired to make the procedure more difficult.

In the "natural cyst" model, the need to overcome two barriers (i.e. the gastric wall and the cyst-forming colonic wall) and ostomy elasticity made it more difficult to puncture since the colonic wall offers a higher resistance to the needle bevel. Although this is a clear disadvantage when learning standard cystoenterostomy, it can be useful to understand some difficulties in dealing with more complex procedures, such as EUSguided biliary drainage, where more than one structure (the gastric or duodenal wall) must be passed. We did not intend to evaluate the ability of the models to teach endoscopic necrosectomy, but obviously both are equally suitable to perform large hydrostatic dilations through the cystoenterostomy tract and undertake removal of any kind of material previously introduced into the colonic pouch or the ostomy bag to emulate walled-off pancreatic necrosis.

In our study, using ultrasound gel prevented leakage from the colonic pouch or the ostomy bag, but the mixture of gel and water created bubbles. Different filling materials, such as gelatin, can be used, but we found ultrasound gel to be easy to use and considered those bubbles as introducing a mild difficulty fairly representative of images found in pseudocysts modified by infection or partial liquefaction.

In conclusion, "natural" and "artificial cysts" are not significantly different in terms of overall satisfaction and realism but the "artificial cyst" appeared to be better in teaching EUCE. Each of these models can be implemented in a structured teaching program in interventional biliopancreatic and endosonographic interventional endoscopy programs, with a preference for the "artificial cyst" model when only EUCE, and not other interventional EUS techniques, such as biliary drainage, is considered. Larger applications of the "artificial cyst" model will help validate it as a standard for training.

\section{Acknowledgements}

We thank all the team from the "surgical school" of Paris and especially Mr. Taleb and Mr. Legagneux for all their assistance. Olympus is also thanked for the loan of endoscopes used during our sessions. 
Competing interests

None

\section{References}

[1] Andersson B, Nilsson E, Willner J et al. Treatment and outcome in pancreatic pseudocysts. Scand J Gastroenterol 2006; 41: 751 - 756

[2] Aghdassi A, Mayerle J, Kraft M et al. Diagnosis and treatment of pancreatic pseudocysts in chronic pancreatitis. Pancreas 2008; 36: $105-$ 112

[3] Fabbri C, Luigiano C, Maimone A et al. Endoscopic ultrasound-guided drainage of pancreatic fluid collections. World J Gastrointest Endosc 2012; 4: 479-488

[4] Ulla-Rocha JL, Vilar-Cao Z, Sardina-Ferreiro R. EUS-guided drainage and stent placement for postoperative intra-abdominal and pelvic fluid collections in oncological surgery. Ther Adv Gastroenterol 2012; 5: $95-102$

[5] Varadarajulu S, Tamhane A, Blakely J. Graded dilation technique for EUS-guided drainage of peripancreatic fluid collections: an assessment of outcomes and complications and technical proficiency (with video). Gastrointest Endosc 2008; 68: 656-666
[6] Ng PY, Rasmussen DN, Vilmann P et al. Endoscopic ultrasound-guided drainage of pancreatic pseudocysts: medium-term assessment of outcomes and complications. Endosc Ultrasound 2013; 2: 199-203

[7] Schöfl R, Buchmeier B, Hauder G. Adaptation of the Erlangen Active Simulator for Interventional Endoscopy (EASIE) model for transmural pancreatic pseudocyst drainage. Endoscopy 2006; 38: 100

[8] Hochberger J, Euler K, Naegel A et al. The compact Erlangen Active Simulator for Interventional Endoscopy: a prospective comparison in structured team-training courses on "endoscopic hemostasis" for doctors and nurses to the "Endo-Trainer" model. Scand J Gastroenterol 2004; 39: 895-902

[9] Maiss J, Wiesnet J, Proeschel A et al. Objective benefit of a 1-day training course in endoscopic hemostasis using the "compactEASIE" endoscopy simulator. Endoscopy 2005; 37: $552-558$

[10] Maiss J, Prat F, Wiesnet J et al. The complementary Erlangen active simulator for interventional endoscopy training is superior to solely clinical education in endoscopic hemostasis - the French training project: a prospective trial. Eur J Gastroenterol Hepatol 2006; 18: $1217-1225$

[11] Baron TH, DeSimio TM. New ex-vivo porcine model for endoscopic ultrasound-guided training in transmural puncture and drainage of pancreatic cysts and fluid collections (with videos). Endosc Ultrasound 2015; 4: $34-39$ 